\title{
ENTEROTOXIGENICIDADE DE Staphylococcus spp. ISOLADOS DE LEITE IN NATURA ${ }^{1}$
}

\author{
Tânia Lúcia Montenegro STAMFORD ${ }^{2, *}$, Celiane Gomes Maia da SILVA \\ Rinaldo Aparecido MOTA* Adelino da CUNHA NETO ${ }^{5}$
}

\begin{abstract}
RESUMO
Os alimentos são passíveis de contaminação por diferentes agentes etiológicos, podendo levar a doenças manifestadas por ação de microorganismos patogênicos ou suas toxinas. Pesquisou-se a ocorrência de cepas de Staphylococcus, assim como a sua capacidade para produção de enterotoxinas em leite produzido e/ou comercializado no Estado de Pernambuco, Brasil. Foram isoladas e selecionadas 109 cepas de Staphylococcus coagulase positiva e negativa de leite in natura. A identificação das cepas isoladas foi realizada por meio de testes morfológicos e bioquímicos, como: testes de catalase, coagulase, hemólise, DNAse, termonuclease, produção de acetoína (VP) e metabolismo de carboidratos (glicose, maltose e manitol). Das 77 cepas coagulase positivas foram identificadas S. aureus (30), S. hyicus (3), S. intermedius (16), S. aureus identificação presuntiva (13) e Estafilococos Coagulase Positiva (SCP) (15). Das 32 cepas coagulase negativa foram identificadas S. capitis (2), S. carnosus (1), S. chromogenes (6), S. hyicus (1), S. schleiferi (1) e Estafilococos Coagulase Negativa (SCN) (21). Foram selecionadas 43 cepas que apresentaram reações de termonuclease evidentes, para análise de enterotoxinas estafilocócicas, realizada pelo teste imunoenzimático ELISA. Os resultados obtidos evidenciaram dez cepas com reação negativa para enterotoxinas: S. aureus (4), S. carnosus (1), S. chromogenes (2), S. hyicus (2) e S. intermedius (1). Entre as cepas enterotoxina positiva, foram encontrados: S. aureus (17), S. chromogenes (2), S. hyicus (1), S. intermedius (8), S. aureus identificado presuntivamente (2), cepas do grupo SCP (1) e as do SCN (2). As espécies que apresentaram maior número de linhagens enterotoxigênicas foram: S. aureus e S. intermedius. Esses resultados podem ser atribuídos à manipulação inadequada do leite e/ou à recontaminação durante o seu armazenamento e distribuição.

Palavras-chave: enterotoxinas, Staphylococcus, alimentos, leite.
\end{abstract}

\section{SUMMARY}

ENTEROTOXIGENICITY OF Staphylococcus spp. ISOLATED OF MILK IN NATURA. Foods are susceptible to contamination for different etiological agents, which can take to manifested diseases by action of pathogenic microorganisms or their toxins. It was researched the occurrence of strains of Staphylococcus and your capacity in producing enterotoxins in milk in natura, that have been produced or commercialized in the State Pernambuco, Brazil. 109 strains of Staphylococus positive and negative coagulase of milk in natura were selected. The identification of the isolated strains was accomplished through morphologic and biochemical tests as: catalase, coagulase, haemolysins, DNAse, thermonuclease, acetoin production (VP) and carbohydrates metabolism (glucose, maltose and mannitol). From the 77 coagulase positive strains 30 were identified as S. aureus, 3 as S. hyicus, 16 as S. intermedius, 13 as S. aureus identification presumptive and 15 as SCP. Among 32 coagulase negative strains 2 were identified as S. capitis, 1 as S. carnosus, 6 as S. chromogenes, 1 as S. hyicus, 1 as S. schleiferi and 21 as SCN. Fourty-three strains that presented very evident thermonuclease reaction, were selected in order to perform for staphylococcal enterotoxins analysis by the immuno enzimatic test (ELFA). 10 strains showed negative reaction for enterotoxins: S. aureus (4), S. carnosus (1), S. chromogenes (2), S. hyicus (2) and S. intermedius (1). Strains that gave positive results, were S. aureus (17), S. chromogenes (2), S. hyicus (1), S. intermedius (8), S. aureus identified presumptively (2) and of the groups SCP (1) and SCN (2). The species that presented larger number of enterotoxigenics strains were $S$. aureus and S. intermedius. Results can be attributed to the inadequate manipulation or food recontamination during the storage and distribution.

Keywords: enterotoxins, Staphylococcus, food, milk.

\section{1 - INTRODUÇÃO}

Os alimentos são passíveis de contaminação por diferentes agentes etiológicos, podendo levar a doenças, manifestadas por ação de microorganismos patogênicos ou

${ }^{1}$ Recebido para publicação em 29/6/2004. Aceito para publicação em 23/1/2006 (001371)

${ }^{2}$ Professora adjunta do Departamento de Nutrição da Universidade Federal de Pernambuco (UFPE)

${ }^{3}$ Doutoranda, mestre em Ciência dos Alimentos pelo programa de pós-graduação em Nutrição da Universidade Federal de Pernambuco (UFPE)

${ }^{4}$ Professor-adjunto do Departamento de Medicina Veterinária da Universidade Federal Rural de Pernambuco (UFRPE)

${ }^{5}$ Professor assistente do Departamento de Nutrição da Universidade Federal de Mato Grosso (UFMT)

Rua Jader de Andrade, 335, Casa Forte

CEP 52061-060, Recife (PE)

E-mail: tlmstamford@yahoo.com.br

*A quem a correspondência deve ser enviada por suas toxinas. Todos os alimentos deveriam ser objeto de exames microbiológicos, que refletiriam as condições higiênicas relacionadas com a produção, armazenamento, transporte e manuseio, a fim de elucidar a ocorrência de enfermidades transmitidas por meio dos alimentos. O leite, independente de sua origem, deve garantir qualidade e inocuidade ao consumidor, devido à sua composição nutricional rica em proteínas e outros componentes [23].

Muitas intoxicações alimentares têm etiologia conhecida, podendo ocorrer nos países em desenvolvimento e nos desenvolvidos. Qualquer tipo de alimento pode dar origem a um surto de intoxicação. Entretanto, os surtos associados a alimentos industrializados têm recebido grande difusão e atenção do público, ao passo que os casos individuais ocorridos em lares, bares e restaurantes são menos relatados ao público devido à falta de notificação ao poder público pela população em geral [4, 8, 21]. 
O S. aureus é um dos agentes patogênicos mais comuns, responsáveis por surtos de intoxicação alimentar. As peculiaridades do seu habitat tornam sua presença largamente distribuída na natureza, sendo transmissíveis aos alimentos por manipuladores, na maioria, portadores assintomáticos, e pelos animais, principalmente o gado leiteiro com mastite [3].

A mastite bovina é considerada um dos principais problemas de ordem infecciosa de bovinos produtores de leite, sendo que as causadas por Staphylococcus aureus são as que mais prevalecem mundialmente. Quanto ao aspecto de saúde pública, a mastite merece importância devido ao risco potencial para transmissão dos microorganismos patógenos ao homem, pelo do leite e derivados. Os prejuízos econômicos causados pela mastite, aliados às questões de saúde pública, tornam a doença, uma das maiores preocupações da Medicina Veterinária por várias razões: epidemiologia abrangente, ser de causa plurietiológica e apresentar controle complexo na dependência dos agentes envolvidos e do meio ambiente no qual os animais são criados [3].

A notificação da intoxicação estafilocócica não é considerada compulsória no Brasil e em diversos países; portanto desconhece-se a verdadeira incidência devido à sintomatologia branda e de curta duração, uma vez que apenas grandes surtos chegam ao conhecimento das autoridades sanitárias [7].

A intoxicação alimentar estafilocócica é atribuída à ingestão de toxinas produzidas e liberadas pela bactéria durante sua multiplicação no alimento, representando um risco para a saúde pública. A toxina é termoestável, podendo permanecer no alimento mesmo após o cozimento, favorecendo a ocorrência da intoxicação [2].

A técnica imunoenzimática que permite a detecção de enterotoxinas estafilocócicas, pelo equipamento mini-vidas, é a Enzyme-linked Fluorescent Assay (ELFA). Essa técnica distingue-se da técnica ELISA na utilização de um leitor da fluorescência desenvolvida durante a análise, reação antígenoanticorpo (com participação de anticorpos policlonais) e uso da enzima fosfatase alcalina [27].

O presente estudo teve como objetivo verificar a ocorrência de cepas de Staphylococcus e a sua capacidade para produção de enterotoxinas em leite in natura.

\section{2 - MATERIAL E MÉTODOS}

Foram selecionadas 109 cepas de Staphylococcus spp., oriundas de leite in natura de vacas com mastite subclínica e procedentes de oito fazendas localizadas no município de Garanhuns, região agreste de Pernambuco.

\section{1 - Métodos}

\subsection{1 - Preparo de diluições das amostras para contagem, isolamento e identificação de Staphylococcus spp.}

As amostras foram preparadas de acordo com CUNHA NETO, SILVA, STAMFORD [6].

\subsection{2 - Provas bioquímicas}

Catalase e Dnase [12]; coagulase [25]; utilização de glicose, maltose e manitol; teste de Voges-ProusKauer segundo a metodologia descrita por MAC FADDIN [16], e crescimento em $\mathrm{NaCl}$ a 7,5\% de acordo com CHAPMAN [5]. Na identificação das espécies coagulase positiva, utilizou-se o teste de acetoína (teste de Voges - Proskauer) em virtude da recomendação pelo Conselho Nacional de Mastite - USA, como sendo um meio adicional para diferenciação de S. aureus das outras cepas coagulase positiva, por esta apresentar um alto grau (90\%) de positividade para acetoína a partir de glicose, enquanto que as cepas de S. intermedius e $S$. hyicus são acetoína negativa [22].

\subsection{3 - Extração e detecção da produção de termonuclease}

A extração da enzima foi realizada pelo método descrito por TATINI et al. (1975), adaptado por HIROOKA, VICENTE, YOSHIMOTO [10]. A detecção da enzima foi realizada pelo método descrito por LACHICA, HOEPRICH, FRANTI [13]. As cepas foram inoculadas em caldo infusão cérebro coração (BHI) e incubadas a $36^{\circ} \mathrm{C}$ por 24 h. Após este período, adicionou-se $500 \mu \mathrm{L}$ de ácido tricloroacético (TCA 3,0 M) à suspensão bacteriana, e submeteu-se à centrifugação a 7.800 rpm por 15 minutos a $4^{\circ} \mathrm{C}$. Desprezou-se o sobrenadante e o precipitado foi ressuspenso em 1,5 mL de tampão TRIS 0,5 M com pH 10,0 adicionado de $1 \%$ de peptona bacteriológica. A atividade nucleásica foi realizada em placas contendo ágar azul de orto-toluidina - DNA, onde foram feitos poços, nos quais foram inoculados com $5 \mu \mathrm{L}$ da mistura (precipitado+tampão TRIS 0,5 M, pH 10,0) previamente aquecida a $100^{\circ} \mathrm{C}$ por 15 min para inativação de nucleases termossensíveis. As placas foram incubadas a $50^{\circ} \mathrm{C}$ por $4 \mathrm{~h}$ e os poços que apresentaram halo de cor rosado foram considerados positivos.

\subsection{4 - Produção e detecção de enterotoxina pelas cepas de Staphylococcus spp.}

\subsubsection{1 - Produção da enterotoxina}

As cepas termonuclease positivas foram ativadas em caldo BHI. Em seguida, alíquota de $2 \mathrm{~mL}$ foi transferida em caldo $\mathrm{BHI}$, enriquecido com $1 \%$ de extrato de levedura (YE). E, posteriormente, incubada a $36^{\circ} \mathrm{C} / 18 \mathrm{~h} / 150 \mathrm{rpm}$, em uma mesa agitadora.

\subsubsection{2 - Purificação da enterotoxina}

Retirou-se uma alíquota de $2 \mathrm{~mL}$ de inóculo do caldo BHI, colocou-se em tubo de filtração/centrifugação (VIDAS tubes SET - bioMérieux Vitek-France) à temperatura de $4^{\circ} \mathrm{C}$ por $15 \mathrm{~min}$ a $3.000 \mathrm{rpm}$.

\subsubsection{3 - Detecção das enterotoxinas}

Após a filtração e centrifugação, retirou-se uma alíquota de $500 \mu \mathrm{L}$ do filtrado, colocou-se no poço do barrete do kit VIDAS SET Staph Enterotoxin, submeteu-se ao exame do leitor de ELISA a $450 \mathrm{~nm}$, mini-VIDAS (mini-VIDAS 
bioMérieux, Inc.France). As amostras que apresentaram títulos de VT (valor do teste) $\leq 0,13$ foram consideradas negativas para presença de enterotoxina e aquelas que apresentaram títulos de VT $>0,13$, consideradas positivas [27]. Sendo o VT a relação entre a fluorescência relativa do padrão e a fluorescência relativa da amostra.

\section{3 - RESULTADOS E DISCUSSÃO}

As 109 cepas de Staphylococcus isoladas de leite in natura foram submetidas ao teste de coagulase e os resultados obtidos mostraram que $71 \%$ das cepas ( 77 amostras) apresentaram positividade (Tabela 1). Resultados semelhantes foram obtidos por ALMEIDA [1], que evidenciou contaminação por Estafilococos Coagulase Positiva em $76,5 \%$ do total de 358 amostras de leite cru oriundas de vacas da região de Garanhuns (PE) com mastite subclínica. LOPES \& STAMFORD [15] ao analisarem 88 cepas de Staphylococcus isoladas de leite cru, detectaram que $54,5 \%$ das amostras foram coagulase positiva.

Quando o animal apresenta mastite clínica ou subclínica, a população de $S$. aureus é predominante tendo em vista que o mesmo faz parte da flora normal do animal [26]. Os valores percentuais encontrados neste trabalho sugerem elevada ocorrência de mastite subclínica por Staphylococcus coagulase positiva no rebanho leiteiro do Estado de Pernambuco.

Das 32 cepas coagulase negativa, visualiza-se que um número elevado de cepas permaneceu com a denominação de Staphylococcus coagulase negativa (SCN), Devido aos testes bioquímicos realizados terem permitido a identificação somente das espécies S. carnosus, S. capitis, S. chromogenes, S. schleiferi e S. hyicus.

$\mathrm{Na}$ coleta de amostras, selecionaram-se os animais portadores de mastite subclínica (Tabela 2), onde pode-se observar o predomínio das espécies coagulase positiva, S. aureus, S. intermedius e S. hyicus, além do grupo classificado como Estafilococos Coagulase Positiva (SCP). Estas representaram aproximadamente $67,8 \%$ das cepas isoladas das amostras de leite analisadas em relação aos $31,9 \%$ de espécies coagulase negativa aqui representadas por S. carnosus, S. chromogenes, S. capitis, S. schleiferi e o grupo classificado como Estafilococos Coagulase Negativa (SCN).

TABELA 2 - Staphylococcus spp. isolados de leite in natura de vacas com mastite subclínica procedentes da região agreste do Estado de Pernambuco

\begin{tabular}{lcc}
\hline Espécies isoladas & $\mathbf{n}^{\circ}$ & $\%$ \\
\hline S. aureus & & \\
S. carnosus & 30 & 27,5 \\
S. capitis & 1 & 0,9 \\
S. chromogenes & 2 & 1,8 \\
S. hyicus & 6 & 5,5 \\
S. intermedius & 4 & 3,6 \\
S. schleiferi & 16 & 14,6 \\
Staphylococcus (SCN) & 1 & 0,9 \\
Staphylococcus (SCP) & 21 & 19,2 \\
S. aureus & 15 & 13,8 \\
Total & 13 & 11,9 \\
\hline S. aureus & 109 & 100 \\
\hline
\end{tabular}

${ }^{1}$ S. aureus - somente com identificação presuntiva. ${ }^{2}$ S. aureus - com identificação confirmativa. SCP - Staphylococcus spp. coagulase positive. SCN - Staphylococcus spp. coagulase negativa

A ocorrência de S. aureus, S. intermedius (cepas coagulase positiva), S. chromogenes e $S$. hyicus em alimentos como leite e derivados é comum, pelo fato de serem encontrados naturalmente na flora de animais como cabras, vacas e ovelhas, além de serem isolados do úbere desses animais com mastite clínica ou subclínica. S. aureus, S. hyicus e S. chromogenes são espécies encontradas com maior freqüência [19]. ROBERSON et al. [22], por meio dos métodos para diferenciação de Staphylococcus spp. coagulase positiva,

TABELA 1 - Comportamento de cepas identificadas de Staphylococcus spp., isoladas de leite in natura, com relação aos testes bioquímicos

\begin{tabular}{|c|c|c|c|c|c|c|c|c|c|c|c|}
\hline & \multirow[b]{2}{*}{$\begin{array}{l}\text { Número } \\
\text { total }\end{array}$} & \multicolumn{10}{|c|}{ Espécies de Staphylococcus } \\
\hline & & aureus $^{1}$ & aureus $^{2}$ & carnosus & capitis & chromogenes & hyicus & intermedius & schleiferi & SCP & SCN \\
\hline & 109 & 13 & 30 & 1 & 2 & 6 & 4 & 16 & 1 & 15 & 21 \\
\hline Coagulase & 77 & 13 & 30 & 0 & 0 & 0 & 3 & 16 & 0 & 15 & 0 \\
\hline $\begin{array}{l}\text { Termo } \\
\text { nuclease }\end{array}$ & 58 & 0 & 30 & 0 & 0 & 6 & 2 & 16 & 1 & 0 & 3 \\
\hline Catalase & 107 & 13 & 30 & 1 & 2 & 6 & 4 & 16 & 1 & 14 & 20 \\
\hline Acetoina & 70 & 10 & 30 & 0 & 2 & 6 & 1 & 0 & 0 & 10 & 11 \\
\hline Manitol & 76 & 8 & 25 & 0 & 2 & 5 & 0 & 14 & 0 & 8 & 14 \\
\hline Maltose & 65 & 12 & 23 & 0 & 0 & 5 & 0 & 15 & 0 & 1 & 9 \\
\hline
\end{tabular}

${ }^{1}$ S. aureus - somente com identificação presuntiva. ${ }^{2}$ S. aureus - com identificação confirmativa. SCP - Staphylococcus spp. coagulase positive. SCN - Staphylococcus spp. coagulase negative. Glicose A - glicose em aerobiose. Glicose $\mathrm{Na}$ - glicose em anaerobiose 
verificaram que a prevalência e patogenicidade de $S$. hyicus e S. intermedius como agente causal de mastite bovina é indeterminada. Os autores sugerem que a utilização de métodos bioquímicos (ex: produção de acetoína), para diferenciação destas linhagens, permite a avaliação da prevalência deste microorganismo como agente causal de mastite.

Os resultados do teste de termonuclease das 109 cepas de Staphylococcus, isoladas de amostras de leite, mostraram positividade em 53\% dos isolados. LOPES \& STAMFORD [15], analisando cepas de Staphylococcus isoladas de leite de vaca cru, verificaram reação de termonuclease positiva em $46 \%$ das cepas. Por outro lado, esses resultados diferem dos obtidos por SILVA, STAMFORD, LIMA [24], que ao analisarem 271 cepas de Staphylococus spp isoladas de amostras de carne de sol, quanto à capacidade de produzirem a enzima termonuclease, verificaram a presença dessa enzima em somente $15 \%$ das cepas.

Levantamentos epidemiológicos realizados por pesquisadores, em fazendas de gado leiteiro no Zimbábue, Israel e Estados Unidos, mostraram percentuais de 10,9; 53; 50 a $78,7 \%$; respectivamente, de prevalência de $S$. aureus em vacas com mastite [11, 18, 28]. Esses dados revelam uma elevada ocorrência de $S$. aureus em leite obtido de animais com mastite [10].

No presente trabalho, isolou-se do leite in natura: S. intermedius, S. schleiferi e S. capitis, sendo as duas primeiras espécies relatadas em cães, no leite de outros animais e isoladas de infecções em humanos [17], não havendo, porém, relato dessas cepas em amostras de leite de vaca, na literatura consultada. O S. capitis é comumente encontrado em tetas e leite de cabra e ovelhas com mastite [4].

Além das cepas de $S$. capitis e $S$. schleiferi, isolou-se do leite in natura: $S$. carnosus, $S$. chromogenes e 21 cepas classificadas como SCN. Este fato reforça a possibilidade das cepas coagulase negativa poderem ser comumente isoladas de leite de animais com mastite. As espécies isoladas com maior freqüência são: S. chromogenes, S. epidermidis, S. saprophyticus, S. xilosus, S. lentus, S. scuri, S. simulans, S. chonii, S. carnosus, S. hominis, S. haemolyticus e S. warneri. A mastite causada pelo Staphylococcus coagulase negativa ocorre com maior freqüência em cabras do que em vacas ou ovelhas [19].

Diante do exposto, torna-se evidente a necessidade de um permanente controle higiênico-sanitário do leite para consumo humano nas etapas de produção, industrialização e comercialização, contribuindo na inocuidade dos alimentos.

A Tabela 1 apresenta o comportamento bioquímico das 109 linhagens isoladas, das quais 58 apresentaram reação positiva para termonuclease; dessas 58 linhagens, 43 cepas com reação típica foram selecionadas para serem submetidas à prova de detecção de enterotoxina. GELLI \& MARTINS [9] destacaram a importância do teste da termonuclease, enzima considerada forte indicadora de presença da enterotoxina estafilocócica em alimentos.
Das 43 cepas de Staphylococcus submetidas ao teste para detecção de enterotoxina, 33 (77\%) foram consideradas positivas, havendo um predomínio de $S$. aureus com identificação presuntiva e confirmatória, em número de 19 cepas positivas e também do $S$. intermedius com nove cepas.

As espécies de Staphylococcus analisadas apresentaram reação positiva para enterotoxinas estafilocócicas em percentuais acima de 50\% (Tabela 3 ), resultados superiores obtidos por outros pesquisadores, sobretudo em amostras de leite. Estes resultados corroboram com LEE et al. [14] quanto à quantidade de S. aureus isolados, produtores de enterotoxinas em bovinos, mostrando que estes podem variar de pequenos intervalos de $5 \%$ até superiores a $30 \%$.

TABELA 3 - Linhagens de Staphylococcus produtoras de enterotoxinas

\begin{tabular}{lccc}
\hline \multirow{2}{*}{ Espécies } & \multicolumn{2}{c}{$\mathbf{n}^{\circ}$ de linhagens analisadas } & \multicolumn{2}{c}{ Linhagens positivas } \\
& & $\mathbf{n}^{\circ}$ & $\%$ \\
\hline S. aureus ${ }^{2}$ & 22 & 17 & 77 \\
S. intermedius & 9 & 8 & 88,9 \\
S. carnosus & 1 & 0 & 0 \\
S. chromogenes & 4 & 2 & 50 \\
S. hyicus & 2 & 1 & 50 \\
S. aureus ${ }^{1}$ & 2 & 2 & 100 \\
SCN & 2 & 2 & 100 \\
SCP & 1 & 1 & 100 \\
\hline${ }^{1}$ S. aureus - somente com identificação presuntiva. ${ }^{2}$ S. aureus - com identificação \\
confirmativa. SCP - Staphylococcus spp. coagulase positive. SCN - Staphylococcus \\
spp. coagulase negativa
\end{tabular}

O gênero Staphylococcus é o agente responsável por aproximadamente $45 \%$ das toxiinfecções no mundo. A contaminação com Staphylococcus spp., pode ocorrer durante os estágios de produção ou estocagem do alimento, por cepas de origem ambiental ou humana. Em condições favoráveis de temperatura, o microorganismo cresce, podendo produzir toxinas. O controle das enterotoxicoses é possível desde que se observem as boas práticas sanitárias de higiene nos estágios de obtenção, produção, estocagem e manuseio de alimentos, pois a proteção contra esta enfermidade é considerada uma obrigação dos profissionais da área de alimentos e de saúde.

\section{4 - CONCLUSÕES}

Os resultados obtidos no presente trabalho permitiram inferir as seguintes conclusões: S. aureus, S. carnosus, S. capitis, S. chromogenes, S. hyicus, S. schleiferi, S. intermedius e $S$. aureus (com identificação presuntiva), além dos grupos de SCN e SCP; podem fazer parte da flora contaminante de leite in natura.

Entre os Staphylococcus, a espécie $S$. aureus é a mais prevalecente para o leite in natura. As espécies $S$. aureus $e$ S. intemedius são as que produzem enterotoxinas com maior freqüência. 


\section{5 - REFERÊNCIAS BIBLIOGRÁFICAS}

[1] ALMEIDA, M.A.C. Prevalência de mastite subclínica em bovinos por Staphylococcus sp. e Streptococcus $s p$. na microrregião de Garanhuns. 199748 p. Mestrado em Medicina Veterinária. Universidade Federal Rural de Pernambuco.

[2] ALCARÃS, L.E.; SATORRES, S.E.; SEPULVEDA, L.; CENTORBI, O. N. P. Detección de Staphylococcus aureus spp. en manipuladores de alimentos. La Alimentación Latino Americana, n. 219, p. 44-47, 1997.

[3] BALABAN, N.; RASOOLY, A. Staphylococcal enterotoxins (review). International Journal of Food Microbiology. v. 61 , p. 1-10, 2000.

[4] BAUTISTA, L.; GAYA, P.; MEDINA, M.; NUÑEZ, M. A quantitative study of enterotoxin production by sheep milk staphylococci. Applied and Environmental Microbiology, v. 59, n. 2, p. 566-569, 1988.

[5] CHAPMAN, G.H. The significance of sodium chloride in studies of staphylococci. Journal of Bacteriology, n. 50, p. 201-203, 1945.

[6] CUNHA NETO, A., SILVA, C.G.M.; STAMFORD, T.L.M. Staphylococcus enterotoxigênicos em alimentos in natura e processados no Estado de Pernambuco, Brasil. Revista Ciência e Tecnologia dos Alimentos, v. 22, n. 3, p. 263-271, 2002.

[7] FRAZIER, W.C.; WESHOFF D.C. Microbiologia de los alimentos. Zaragoza, España, 2000.

[8] GELLI, D.S.; JACABI, M.; SAKUMA, H.; RAMALHO, A.M.; RISTORI, C.A. et al. Surtos de enfermidades transmitidas por alimentos (ETAs) investigados pelos laboratórios de saúde pública do Estado de São Paulo, no período de 1994 a 1998. XX Congresso Brasileiro de Microbiologia, Salvador (BA), 1999.

[9] GELLI, D.S.; MARTINS, M.C. Staphylococcus aureus produtor de termonuclease em alimentos. Revista do Instituto Adolfo Lutz, v. 46, n. 1/2, p. 103-109, 1986.

[10] HIROOKA, E.Y.; VICENTE, E.; YOSHIMOTO, Y. Detecção de termonuclease estafilocócica em leite: extração e manutenção da atividade enzimática. Revista Ciência e Tecnologia de Alimentos, v. 10, n. 2, p. 231-248, 1990.

[11] HOROVITZ, C.T.; ZIV, G. Susceptibility of Staphylococcus aureus of bovine udder origin to antimicrobial drugs and heavy metal. Israel Journal of Veterinary Medicine, v. 44, n. 2, p. 119-123, 1988.

[12] KONEMAN, E.W.; ALLEN, S.D.; JANDA, W.M.; SCHRECKERNBERGER, P.C.; WINN, W.C. Diagnóstico Microbiológico - Texto y Atlas Color. Buenos Aires, Editorial Médica Panamericana; 1999.

[13] LACHICA, R.V.F.; HOEPRICH, P.D.; FRANTI, C.E. Convenient assay for staphylococcal nuclease by the metachromatic well-agar-diffusion technique. Applied Microbiology. Washington, v. 24, n. 6, p. 920-923, 1972.

[14] LEE, S.U et al. Characterization of staphylococcal bovine mastitis isolates using the polymerase chain reaction. Journal of Food Protection, v. 61, n. 10, p. 1384-1386, 1998.

[15] LOPES, A.C.S.; STAMFORD, T.L.M. Pontos críticos de controle no fluxograma de beneficiamento do leite pasteurizado. Arquivos Latinoamericanos de Nutrición, v. 47, n. 43, p. 67-371, 1997.
[16] MAC FADIN, J.F. Pruebas bioquímicas para la identificatión de bacterias de importancia clinica. Buenos Aires, Argentina, 1973.

[17] MAHOUDEAU, I.; DELABRANCHE, X.; PREVOST, G.; MONTEIL, H.; PIEMONT, Y.V.E.S. Frequency of isolation of Staphylococcus intermedius from humans. Journal of Clinical Microbiology, v. 35, n. 8, p. 2153-2154, 1997.

[18] MAKAYA, P.V.; AASRESTRUP, F.M.; OLSEN J.E. Distribution and antibiotic resistance paterns of common mastitis pathogens (gram-positive cocci) in selected dairy herds of three farmings sectors in Zimbabwe. Zimbabwe Veterinary Journal, v. 27, n. 2, p. 65-72, 1996.

[19] NICKERSON, S.C.; OWENS, W. E.; BODDIE, R.L. Mastitis in dairy heifers: initial studies on prevalence and control. Journal of Food Protection, v. 78, n. 7, p. 1607-1618, 1995.

[20] NOTERMANS, S.; BORGDORFF, M.A global perspective of foodborne disease. Journal of Food Protection, v. 60, n. 11, p. 1395-1399, 1997.

[21] PASSOS, M.H.C.R.; KUAYE, A.Y. Relato de surtos de intoxicação alimentar provocada por consumo de bolo contaminado por Staphylococcus aureus - importância da higiene dos manipuladores e condições de conservação do alimento na prevenção da doença. Revista do Instituto Adolfo Lutz, v. 56, n. 1, p. 71-76, 1996.

[22] ROBERSON, J. R.; FOX, L. K.; HANCOCK, D. D.; BESSER, T. E. Evaluation of methods for differentiation of coagulase-positive staphylococci. Journal of Clinical Microbiology, v. 30, n. 12, p. 3.217-3.219, 1992.

[23] SENA, M.J. Perfil epidemiológico, resistência a antibióticos e aos conservantes nisina e sistema lactoperoxidase de Staphylococcus $s p$. isolados de queijos coalho comercializados em Recife (PE). 1997 75 p. Tese de doutorado. Escola de Veterinária, Universidade Federal de Minas Gerais (UFMG), 2000.

[24] SILVA, M.C.D. da; STAMFORD, T.L.M.; LIMA, A. W.O. Condições higiênico-sanitárias da carne de sol comercializada no município do Recife (PE). II. Staphylococcus aureus enterotoxigênicos. Arquivos de Biologia e Tecnologia, v. 35, n. 2, p. 375-388, 1992.

[25] SPERBER, W.zH.; TATINI, S.R. Interpretation of the tube coagulase test for identification of Staphylococcus aureus. Applied Microbiology, v. 29, n. 4, p. 502-505, 1975.

[26] TAKEUCHI, S.; ISHIGURO, K.; IKEGAMI, M. et al. Production of toxic shock syndrome toxin by Staphylococcus aureus isolated from mastitic cow's milk and farm bulk milk. Vet. Microbiol., v. 59, p. 251-258, 1998.

[27] VERNOZY-ROZAND, C.; MEYRAND, A.; DELIGNETTEMULLER, M.L.; JAUBEERT, G.; PERRIN, G.; LAPEYRE; RICHARD, Y. Behaviour and enterotoxin production by Staphylococcaus aureus during the manufacture and ripening of raw goat's milk lactic cheeses. Journal Dairy Reserch, v. 2, p. 273-281, 1998.

[28] WILSON, D.J.; GONZALEZ, N.R.; SEARS, P.M. Secregation or use of separate milking units for cows infected with Staphylococcus aureus: effects on prevalence of infection and bulk tank somatic cell count. Journal Dairy Science, v. 78, n. 9, p. 2083-2085, 1995. 